\title{
An Optimal Procedure for Determining the Height Parameters of Fracture Surfaces
}

\author{
T. Ficker
}

\begin{abstract}
This paper presents an attempt to find an optimal procedure for determining the height parameters of fracture surfaces. This is a useful task that may significantly increase the reliability of topographic analyses of solids. The paper focuses on seeking an optimum number of measuring sites to ensure sufficient reliability of the resulting height parameters determined by the confocal technique. The statistical tests show that the number may be close to 25 measuring sites.
\end{abstract}

Keywords: profile analysis, 3D profile parameters, fracture surfaces, cement-based materials, confocal microscopy.

\section{Introduction}

In fracture mechanics there has been long-term interest in the topographic properties of fracture surfaces. It is believed that fracture surfaces bear valuable information not only on the structure but also on the mechanical properties of solids.

Software analysis of surface topography requires digital replicas of the surfaces to be available. Among several techniques available for this purpose, confocal microscopy is very convenient, since it produces three-dimensional replicas without any contact with the surface, i.e. without any mechanical damage to the specimens. Confocal replicas are reconstructed from a series of horizontal sections, which are in fact digital two-dimensional microscopic images.

As soon as the digital three-dimensional surface relief is formed, software analysis of the surface can begin, and it can reveal various useful surface quantities, including the 3D-profile and 3D-roughness parameters, which are often employed for classifying surface height irregularities [1-4]. These parameters are closely related to structural parameters such as porosity [5], or to mechanical properties, including compressive strength [6].

The 3D-profile parameters $H_{a}, H_{q}$ computed by means of the reconstructed surface profile $f(x, y)$ within the plane rectangle $L \times M$

$$
\begin{aligned}
H_{a} & =\frac{1}{L \cdot M} \iint_{(L M)}|f(x, y)| \mathrm{d} x \mathrm{~d} y \\
H_{q} & =\sqrt{\frac{1}{L \cdot M} \iint_{(L M)}[f(x, y)]^{2} \mathrm{~d} x d y}
\end{aligned}
$$

were tested previously $[1,5,6]$, and were shown to be reliable indicators of both the porosity and the compressive strength of hydrated cement materials. However, the determination of parameters $H_{a}, H_{q}$ is connected with certain particularities that should be borne in mind when they are determined. The first problem is that we do not know the optimal number of surface sites to be visited with the confocal microscope in order to obtain reliable profile parameter values. To resolve this problem, we performed a large series of measurements on a particular fracture surface and used statistical considerations to infer a sufficient number of site measurements to ensure precise results. This paper reports on the whole procedure.

\section{Experiment}

The fracture surface of hydrated cement paste (Fig. 1) was tested. The test specimen was prepared from ordinary Portland cement CEM 42,5 I R-sc. The water-to-cement ratio was set to 0.5 and the period of hydration was one year, i.e. the specimen was of high maturity.

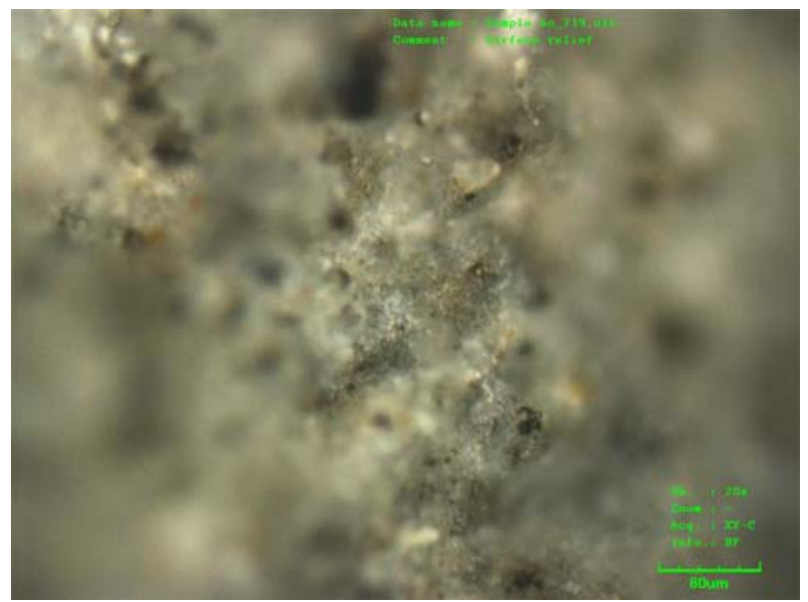

Fig. 1: The surface of a fractured specimen of hydrated cement paste 


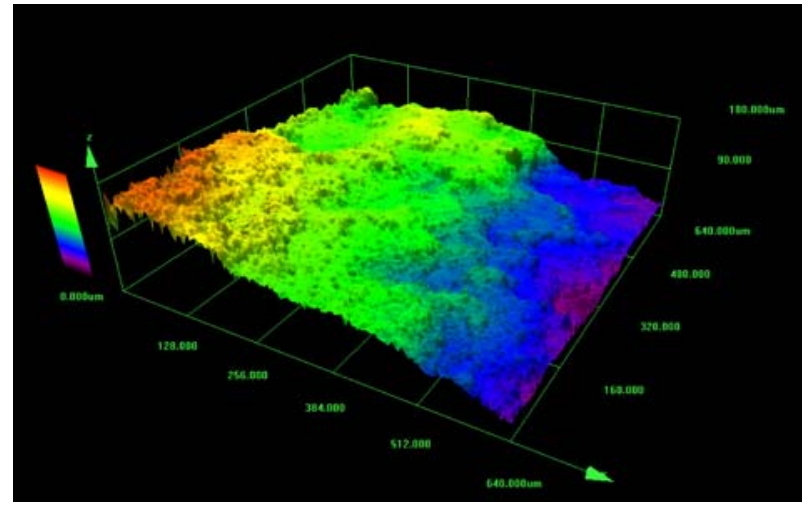

Fig. 2: Confocal relief of a fractured surface

The 3D profiles $f(x, y)$ were created using an Olympus Lext 3100 confocal microscope. One of these profiles is shown in Figure 2. The profiles were formed by the software, which processed a series of optical sections taken at various heights of the fracture surface. Approximately 200 image sections were taken (magnification $20 \times$ ) for each measured surface site, starting from the very bottom of the surface depressions (valleys) and processing to the very top of the surface protrusions (peaks) with a step of $1.28 \mu \mathrm{m}$. The investigated area $L \times M=$ $1280 \mu \mathrm{m} \times 1280 \mu \mathrm{m}(1024$ pixels $\times 1024$ pixels $)$ was chosen in 280 surface sites. These measuring sites consisted of seven groups, i.e. 5, 10, 15, 25, 50, 75 and 100 measuring sites located randomly on the surface. For each group of measurements, parameters $H_{a}, H_{q}$ were computed and their averages were determined. In this way we obtained seven couples of average values, whose statistical reliabilities increased with increasing number of measurements. Naturally, the group of 100 measurements yielded the most reliable averages, and they were therefore adopted as reference values $H_{a}^{(100)}, H_{q}^{(100)}$ of high precision close to exact values. The averages $H_{a}^{(N)}$, $H_{q}^{(N)}$ of the remaining groups $N=5,10,15,50$, and 75 were then classified according to their percentage deviation from the precise reference values

$$
\begin{aligned}
& P_{a}(N)=\frac{\left|H_{a}^{(100)}-H_{a}^{(N)}\right|}{H_{a}^{(100)}} \times 100(\%) \\
& P_{q}(N)=\frac{\left|H_{q}^{(100)}-H_{q}^{(N)}\right|}{H_{q}^{(100)}} \times 100(\%)
\end{aligned}
$$

As the optimal number of measuring sites $N$, we chose a number which ensured that the percentage deviation will be less than five, i.e. $P(N)<5 \%$.

\section{Results and discussion}

In Figure 3, there are two graphs $P_{a}(N)$ and $P_{q}(N)$ showing the behavior of percentage deviations from the reference average values $H_{a}^{(100)}, H_{q}^{(100)}$. Both graphs clearly indicate the optimum number of measuring sites to be close to $N=25$. This number of measuring sites ensures that the percentage deviation is about $5 \%$, which is a normal laboratory statistical deviation. For larger $N>25$, the resulting profile parameters $H_{a}^{(N)}, H_{q}^{(N)}$ would show still lower statistical uncertainty but at the expense of an enormous measuring and computational effort. On the other hand, results with $N<25$ rapidly increase their statistical uncertainty. For example, at $N=5$ the percentage deviation reaches a value of almost $25 \%$, which indicates rather high uncertainty and low reliability.

\section{Conclusion}

The statistical tests presented in this paper have shown that the optimum number of measuring surface sites to determine 3D-profile parameters using the confocal microscopic technique is close to 25 sites in order to ensure sufficient reliability of numerical results. In practice, this means that the measurements may be performed within a matrix of $5 \times 5$ surface points uniformly distributed over the tested surface.

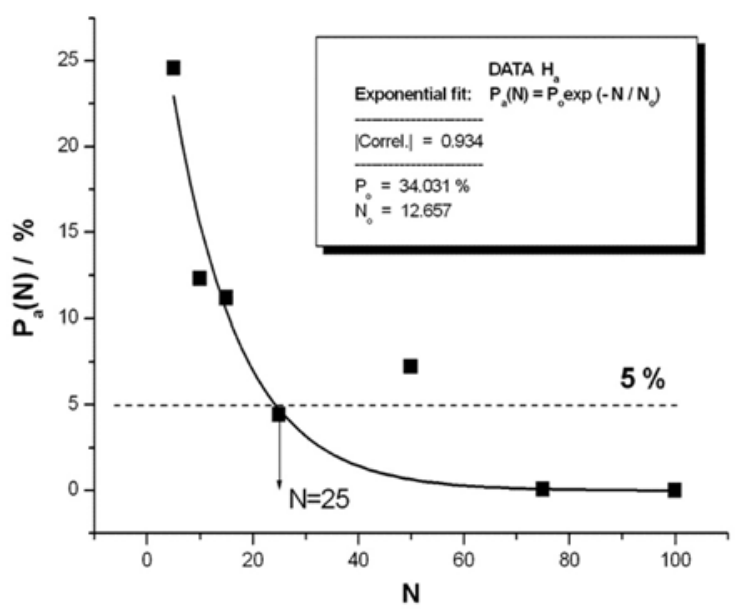

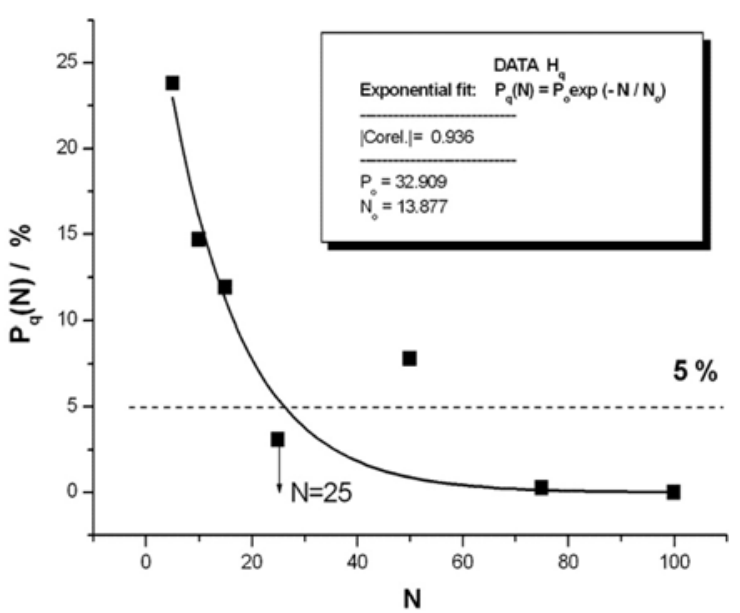

Fig. 3: Percentage deviations of average profile parameters from the reference value representing 100 site measurements 


\section{Acknowledgement}

This work was supported by the Ministry of Education, Youth and Sports of the Czech Republic under Contract no. ME 09046 (Kontakt).

\section{References}

[1] Ficker, T., Martišek, D., Jennings, H. M.: Roughness of fracture surfaces and compressive strength of hydrated cement pastes, Cem. Conr. Res. 40, 2010, 947-955.

[2] Qu, J., Stih, A. J.: Analytical surface roughness parameters of a theoretical profile consisting of elliptical arcs, Machining Science and Technology, 7, 2003, 281-294.

[3] Hong, E.-S, Lee, J.-S., Lee, I.-M.: Underestimation of roughness in rough rock joints, Int. J. $\mathrm{Nu}$ mer. Anal. Meth. Geomech. 32, 2008, 1385-1 403.
[4] Fardin, N., Stephansson, O., Jing, L.: The scale dependence of rock joint surface roughness, International Journal of Rock Mechanics $\&$ Mining Sciences, 38, 2001, 659-669.

[5] Ficker, T., Martišek, D., Jennings, H. M.: Roughness and porosity of hydrated cement pastes, Acta Polytechnica, 51, 2011, 7-20.

[6] Ficker, T.: Fracture surfaces of porous materials, Acta Polytechnica, 51, 2011, 21-24.

Prof. RNDr. Tomáš Ficker, DrSc.

Phone: +420 541147661

E-mail: ficker.t@fce.vutbr.cz

Department of Physics

Faculty of Civil Engineering

Brno University of Technology

Veveří 95, 66237 Brno, Czech Republic 